
Zhou

\title{
Nonlocal Initial Value Problem for Hybrid Generalized Hilfer-type Fractional Implicit Differential Equations
}

https://doi.org/10.1515/msds-2020-0127

Received February 9, 2021; accepted March 30, 2021

\begin{abstract}
In this paper, we prove some existence results of solutions for a class of nonlocal initial value problem for nonlinear fractional hybrid implicit differential equations under generalized Hilfer fractional derivative. The result is based on a fixed point theorem on Banach algebras. Further, examples are provided to illustrate our results.
\end{abstract}

Keywords: Generalized Hilfer fractional derivative, initial value problem, nonlocal, existence, hybrid fractional differential equations, implicit differential equations, fixed point

MSC: 34A08, 26A33

\section{Introduction}

Fractional calculus is a branch of classical mathematics, which is concerned with the generalization of the integer order differentiation and integration of a function to non-integer order, its is a solid and growing field both in theory and in its applications [2-4, 15, 32]. In the last few decades, fractional differentiation and fractional integration have found many applications in various fields of science and engineering. There are numerous kinds of fractional derivatives, such as, Riemann-Liouville fractional derivative, Caputo fractional derivative, Hilfer fractional derivative, Hadamard fractional derivative, Erdélyi-Kober, Katugampola and others; see [1, 5-8, 10, 11, 14, 21, 26-29] and the references therein. For some recent applications, see [22, 23, 25].

Another interesting class of problems involves hybrid fractional differential equations and has received attention of several researchers [9, 12, 19, 31].

In [13], the authors discussed the following terminal value problem for fractional differential equations with generalized Hilfer fractional derivative :

$$
\left\{\begin{array}{l}
\left({ }^{\alpha} \mathbb{D}_{a^{+}}^{9, r} x\right)(t)=f\left(t, x(t),\left({ }^{\alpha} \mathbb{D}_{a^{+}}^{9, r} x\right)(t)\right), \quad t \in I:=[a, T], a>0, \\
\chi(T)=c \in \mathbb{R} .
\end{array}\right.
$$

Their reasoning is mainly based upon different types of classical fixed point theory such as the Banach contraction principle and the Krasnoselskii fixed point theorem.

\footnotetext{
Abdelkrim Salim, Jamal Eddine Lazreg: Laboratory of Mathematics, Djillali Liabes University of Sidi Bel-Abbes, P.0. Box 89 Sidi Bel Abbes 22000, Algeria, E-mail: salimkarim24@yahoo.fr, lazregjamal@yahoo.fr

${ }^{\star}$ Corresponding Author: Mouffak Benchohra: Laboratory of Mathematics, Djillali Liabes University of Sidi Bel-Abbes, P.O. Box 89 Sidi Bel Abbes 22000, Algeria, E-mail: benchohra@yahoo.com

Juan J. Nieto: Departamento de Estatistica, Análise Matemática e Optimización, Instituto de Matemáticas, Universidade de Santiago de Compostela, Santiago de Compostela, Spain, E-mail: juanjose.nieto.roig@usc.es

Yong Zhou: Faculty of Mathematics and Computational Science, Xiangtan University, E-mail: yzhou@xtu.edu.cn
} 
Using Krasnoselskii, Schaefer and Schauder fixed point theorems, Wang and Zhang [30] proved some existence results for the following nonlocal initial value problem for differential equations involving Hilfer fractional derivative :

$$
\left\{\begin{array}{l}
D_{a^{+}}^{g, r} u(t)=f(t, u(t)), \quad t \in(a, b] \\
\left(I_{a^{+}}^{1-\xi} u\right)\left(a^{+}\right)=\sum_{i=1}^{m} \lambda_{i} u\left(\tau_{i}\right)
\end{array}\right.
$$

Derbazi et al. [16] studied the existence and uniqueness of solutions of the following three-point boundary value problem for fractional hybrid differential equations with Caputo fractional derivative :

$$
\left\{\begin{array}{l}
{ }^{c} \mathcal{D}_{0^{+}}^{g}\left(\frac{u(t)-f(t, u(t))}{g(t, u(t))}\right)=h(t, u(t)), t \in J_{1}:=[0, T], \\
a_{1}\left(\frac{u(0)-f(0, u(0))}{g(0, u(0))}\right)+b_{1}\left(\frac{u(T)-f(T, u(T))}{g(T, u(T))}\right)=c_{1}, \\
a_{2}{ }^{c} \mathcal{D}_{0^{+}}^{\beta}\left(\frac{u(t)-f(t, u(t))}{g(t, u(t))}\right)_{t=\eta}+b_{2}{ }^{c} \mathcal{D}_{0^{+}}^{\beta}\left(\frac{u(t)-f(t, u(t))}{g(t, u(t))}\right)_{t=T}=c_{2} .
\end{array}\right.
$$

The proved results rely on a hybrid fixed point theorem for a sum of three operators due to Dhage.

Motivated by the works of the papers mentioned above, we establish in this paper, existence results to the nonlocal initial value problem (IVP for short) with nonlinear implicit hybrid generalized Hilfer type fractional differential equation :

$$
\begin{gathered}
{ }^{\alpha} \mathbb{D}_{a^{+}}^{9, r}\left(\frac{x(t)-\chi(t, x(t))}{f(t, x(t))}\right)=\varphi\left(t, x(t),{ }^{\alpha} \mathbb{D}_{a^{+}}^{9, r}\left(\frac{x(t)-\chi(t, x(t))}{f(t, x(t))}\right)\right), t \in(a, b], \\
\left({ }^{\alpha} \mathbb{J}_{a^{+}}^{1-\xi}\left(\frac{x(\tau)-\chi(t, x(t))}{f(\tau, x(\tau))}\right)\right)\left(a^{+}\right)=\sum_{i=1}^{m} c_{i}\left(\frac{x\left(\epsilon_{i}\right)-\chi\left(\epsilon_{i}, x\left(\epsilon_{i}\right)\right)}{f\left(\epsilon_{i}, x\left(\epsilon_{i}\right)\right)}\right),
\end{gathered}
$$

where ${ }^{\alpha} \mathbb{D}_{a^{+}}^{9, r}{ }^{\alpha} \mathbb{J}_{a^{+}}^{1-\xi}$ are the generalized Hilfer fractional derivative of order $\vartheta \in(0,1)$ and type $r \in[0,1]$ and generalized fractional integral of order $1-\xi,(\xi=\vartheta+r-\vartheta r)$ respectively, $c_{i}, i=1, \ldots, m$, are real numbers, $\epsilon_{i}, i=1, \ldots, m$, are pre-fixed points satisfying $a<\epsilon_{1} \leq \ldots \leq \epsilon_{m}<b, f \in C([a, b] \times \mathbb{R}, \mathbb{R} \backslash\{0\})$, $\chi \in C([a, b] \times \mathbb{R}, \mathbb{R}), \varphi \in C\left([a, b] \times \mathbb{R}^{2}, \mathbb{R}\right)$. Further details and definitions are given in Section 2 .

The present paper is organized as follows. In Section 2, some notations are introduced and we recall some preliminaries about generalized Hilfer fractional derivative and auxiliary results. In Section 3, an existence result for the problem (1)-(2) is presented which is based on a fixed point theorem in Banach algebras [17, 18]. Finally, in the last section, we give an example to illustrate the applicability of our results.

\section{Preliminaries}

In this section, we introduce some preliminary facts, essential notations, definitions and results which are used throughout this paper. Let $0<a<b, J=[a, b]$, and

$$
C_{\xi, \alpha}(J)=\left\{x:(a, b] \rightarrow \mathbb{R}: \text { the function } t \rightarrow \Psi_{\xi}(t, a) x(t) \in C(J, \mathbb{R})\right\}, 0 \leq \xi<1,
$$

where

$$
\Psi_{\xi}(t, a)=\alpha^{\xi-1}\left(t^{\alpha}-a^{\alpha}\right)^{1-\xi},
$$

and

$$
\begin{aligned}
& C_{\xi, \alpha}^{n}(J)=\left\{x \in C^{n-1}(J): x^{(n)} \in C_{\xi, \alpha}(J)\right\}, n \in \mathbb{N}, \\
& C_{\xi, \alpha}^{0}(J)=C_{\xi, \alpha}(J) .
\end{aligned}
$$


with the norm

$$
\|x\|_{C_{\xi, \alpha}}=\sup _{t \in J}\left|\Psi_{\xi}(t, a) x(t)\right| .
$$

Consider the space $X_{c}^{p}(a, b),(c \in \mathbb{R}, 1 \leq p \leq \infty)$ of those complex-valued Lebesgue measurable functions $f$ on $[a, b]$ for which $\|f\|_{X_{c}^{p}}<\infty$, where the norm is defined by

$$
\|f\|_{X_{c}^{p}}=\left(\int_{a}^{b}\left|t^{c} f(t)\right|^{p} \frac{d t}{t}\right)^{\frac{1}{p}}, \quad(1 \leq p<\infty, c \in \mathbb{R}) .
$$

In particular, when $c=\frac{1}{p}$, the space $X_{c}^{p}(a, b)$ coincides with the $L^{p}(a, b)$ space: $X_{\frac{1}{p}}^{p}(a, b)=L^{p}(a, b)$.

Definition 2.1. [20] Let $\vartheta \in \mathbb{R}_{+}, c \in \mathbb{R}$ and $h \in X_{c}^{p}(a, b)$. The generalized fractional integral of order $\vartheta$ is defined by

$$
\left({ }^{\alpha} \mathbb{J}_{a^{+}}^{9} h\right)(t)=\int_{a}^{t} \tau^{\alpha-1} \bar{\Psi}_{\vartheta}(t, \tau) h(\tau) d \tau, t>a, \alpha>0,
$$

where

$$
\bar{\Psi}_{\vartheta}(t, \tau)=\frac{\alpha^{1-\vartheta}}{\Gamma(\vartheta)}\left(t^{\alpha}-\tau^{\alpha}\right)^{9-1}
$$

If $h(t)=1$, we obtain

$$
\begin{aligned}
\left({ }^{\alpha} \mathbb{J}_{a^{+}}^{9} h\right)(t) & =\int_{a}^{t} \tau^{\alpha-1} \bar{\Psi}_{\vartheta}(t, \tau) d \tau \\
& =\frac{1}{\Gamma(\vartheta+1)}\left(\frac{t^{\alpha}-a^{\alpha}}{\alpha}\right)^{\vartheta} \\
& =\bar{\Psi}_{\vartheta+1}(t, a) .
\end{aligned}
$$

Definition 2.2. [20] Let $\vartheta \in \mathbb{R}_{+} \backslash \mathbb{N}$ and $\alpha>0$. The generalized fractional derivative ${ }^{\alpha} \mathbb{D}_{a^{+}}^{9}$ of order $\vartheta$ is defined by

$$
\begin{aligned}
\left({ }^{\alpha} \mathbb{D}_{a^{+}}^{9} h\right)(t) & =\delta_{\alpha}^{n}\left({ }^{\alpha} \mathbb{J}_{a^{+}}^{n-9} h\right)(t) \\
& =\left(t^{1-\alpha} \frac{d}{d t}\right)^{n} \int_{a}^{t} \tau^{\alpha-1} \bar{\Psi}_{n-\vartheta}(t, \tau) h(\tau) d \tau, t>a, \alpha>0,
\end{aligned}
$$

where $n=[\vartheta]+1$ and $\delta_{\alpha}^{n}=\left(t^{1-\alpha} \frac{d}{d t}\right)^{n}$.

Lemma 2.3. $[20,24]$ Let $\vartheta>0,0 \leq \xi<1$. Then, ${ }^{\alpha} \mathbb{J}_{a^{+}}^{9}$ is bounded from $C_{\xi, \alpha}(J)$ into $C_{\xi, \alpha}(J)$.

Lemma 2.4. [24] Let $\vartheta>0,0 \leq \xi<1$, and $h \in C_{\xi, \alpha}(J)$. Then,

$$
\left({ }^{\alpha} \mathbb{D}_{a^{+}}^{9 \alpha} \mathbb{J}_{a^{+}}^{9} h\right)(t)=h(t) .
$$

Lemma 2.5. [24] Let $0<\vartheta<1,0 \leq \xi<1$. If $h \in C_{\xi, \alpha}(J)$ and ${ }^{\alpha} \mathbb{J}_{a^{+}}^{1-9} h \in C_{\xi, \alpha}^{1}(J)$, then

$$
\left({ }^{\alpha} \mathbb{J}_{a^{+}}^{9}{ }^{\alpha} \mathbb{D}_{a^{+}}^{9} h\right)(t)=h(t)-\bar{\Psi}_{\vartheta}(t, a)\left({ }^{\alpha} \mathbb{J}_{a^{+}}^{1-9} h\right)(a), \text { for all } t \in(a, b] .
$$

Definition 2.6. [24] Let order 9 and type $r$ satisfy $n-1<\vartheta<n$ and $0 \leq r \leq 1$, with $n \in \mathbb{N}$. The generalized Hilfer type fractional derivative with $\alpha>0$ of a function $h \in C_{\xi, \alpha}(J)$, is defined by

$$
\left({ }^{\alpha} \mathbb{D}_{a^{+}}^{9, r} h\right)(t)=\left({ }^{\alpha} \mathbb{J}_{a^{+}}^{r(n-9)}\left(t^{\alpha-1} \frac{d}{d t}\right)^{n}{ }^{\alpha} \mathbb{J}_{a^{+}}^{(1-r)(n-9)} h\right)(t)
$$




$$
=\left({ }^{\alpha} \mathbb{J}_{a^{+}}^{r(n-\vartheta)} \delta_{\alpha}^{n \alpha} \mathbb{J}_{a^{+}}^{(1-r)(n-\vartheta)} h\right)(t)
$$

Property 2.7. [24] The operator ${ }^{\alpha} \mathbb{D}_{a^{+}}^{9, r}$ can be written as

$$
{ }^{\alpha} \mathbb{D}_{a^{+}}^{\vartheta, r}={ }^{\alpha} \mathbb{J}_{a^{+}}^{r(1-\vartheta)} \delta_{\alpha}^{\alpha} \mathbb{J}_{a^{+}}^{1-\xi}={ }^{\alpha} \mathbb{J}_{a^{+}}^{r(1-\vartheta) \alpha} \mathbb{D}_{a^{+}}^{\xi},
$$

where $0 \leq r \leq 1$ and $\xi=\vartheta+r-\vartheta r$.

Consider the following parameters $\vartheta, r, \xi$ satisfying

$$
\xi=\vartheta+r-\vartheta r, \quad 0<\vartheta, r, \xi<1 .
$$

We define the spaces

$$
C_{\xi, \alpha}^{\vartheta, r}(J)=\left\{x \in C_{\xi, \alpha}(J),{ }^{\alpha} \mathbb{D}_{a^{+}}^{\vartheta, r} x \in C_{\xi, \alpha}(J)\right\},
$$

and

$$
C_{\xi, \alpha}^{\xi}(J)=\left\{x \in C_{\xi, \alpha}(J),{ }^{\alpha} \mathbb{D}_{a^{+}}^{\xi} x \in C_{\xi, \alpha}(J)\right\} .
$$

Since ${ }^{\alpha} \mathbb{D}_{a^{+}}^{9, r} x={ }^{\alpha} \mathbb{J}_{a^{+}}^{r(1-9) \alpha} \mathbb{D}_{a^{+}}^{\xi} x$, it follows from Lemma 2.3 that

$$
C_{\xi, \alpha}^{\xi}(J) \subset C_{\xi, \alpha}^{\vartheta, r}(J) \subset C_{\xi, \alpha}(J)
$$

Lemma 2.8. [24] If $x \in C_{\xi, \alpha}^{\xi}(J)$, then

$$
\alpha \mathbb{J}_{a^{+}}^{\xi} \alpha \mathbb{D}_{a^{+}}^{\xi} \chi=\alpha \mathbb{J}_{a^{+}}^{\vartheta} \alpha \mathbb{D}_{a^{+}}^{\vartheta, r} x,
$$

and

$$
{ }^{\alpha} \mathbb{D}_{a^{+}}^{\xi}{ }^{\alpha} \mathbb{J}_{a^{+}}^{\vartheta} \chi={ }^{\alpha} \mathbb{D}_{a^{+}}^{r(1-\vartheta)} \chi
$$

Lemma 2.9. [17] Let $B$ be a closed, convex, bounded and nonempty subset of a Banach algebra $(X,\|\cdot\|)$, and let $\mathcal{P}, \mathcal{R}: X \rightarrow X$ and $\mathcal{Q}: B \rightarrow X$ be three operators such that

1) $\mathcal{P}$ and $\mathcal{R}$ are Lipschitzian with Lipschitz constants $\eta_{1}$ and $\eta_{2}$, respectively,

2) $Q$ is compact and continuous,

3) $x=\mathcal{P} x Q y+\mathcal{R} x \Rightarrow x \in B$ for all $y \in B$,

4) $\eta_{1} \beta+\eta_{2}<1$, where $\beta=\|\mathcal{Q}(B)\|=\sup \{\|\mathcal{Q}(y)\|: y \in B\}$.

Then the operator equation $\mathcal{P} x Q x+\mathcal{R} x=x$ has a solution in $B$.

\section{Existence of Solutions}

Let $v: J \rightarrow \mathbb{R}$ be a function such that $v(\cdot) \in C_{\xi, \alpha}(J), f \in C(J \times \mathbb{R}, \mathbb{R} \backslash\{0\})$, and the function $\chi \in C(J \times \mathbb{R}, \mathbb{R})$. We consider the following simpler fractional differential equation related to (1)-(2) with $\varphi \equiv v$.

$$
{ }^{\alpha} \mathbb{D}_{a^{+}}^{9, r}\left(\frac{\chi(t)-\chi(t, \chi(t))}{f(t, \chi(t))}\right)=v(t), \quad t \in(a, b],
$$

where $0<\vartheta<1,0 \leq r \leq 1, \alpha>0$, with the nonlocal condition

$$
\left({ }^{\alpha} \mathbb{J}_{a^{+}}^{1-\xi}\left(\frac{\chi(\tau)-\chi(t, \chi(t))}{f(\tau, \chi(\tau))}\right)\right)\left(a^{+}\right)=\sum_{i=1}^{m} c_{i}\left(\frac{\chi\left(\epsilon_{i}\right)-\chi\left(\epsilon_{i}, \chi\left(\epsilon_{i}\right)\right)}{f\left(\epsilon_{i}, \chi\left(\epsilon_{i}\right)\right)}\right),
$$


where $\xi=\vartheta+r-\vartheta r, c_{i}, i=1, \ldots, m$, are real numbers, $f \in C(J \times \mathbb{R}, \mathbb{R} \backslash\{0\}), \chi \in C(J \times \mathbb{R}, \mathbb{R}), \epsilon_{i}, i=1, \ldots, m$, are pre-fixed points satisfying $a<\epsilon_{1} \leq \ldots \leq \epsilon_{m}<b, v \in C_{\xi, \alpha}(J)$ and $\sum_{i=1}^{m} c_{i} \bar{\Psi}_{\xi}\left(\epsilon_{i}, a\right) \neq 1$. The following theorem shows that the problem (3)-(4) have a solution given by

$$
x(t)=f(t, x(t))\left[\bar{\Psi}_{\xi}(t, a) \frac{\sum_{i=1}^{m} c_{i}\left({ }^{\alpha} \mathbb{J}_{a^{+}}^{9} v(\tau)\right)\left(\epsilon_{i}\right)}{1-\sum_{i=1}^{m} c_{i} \bar{\Psi}_{\xi}\left(\epsilon_{i}, a\right)}+\left({ }^{\alpha} \mathbb{J}_{a^{+}}^{9} v(\tau)\right)(t)\right]+\chi(t, x(t)) .
$$

Theorem 3.1. The function $x$ satisfies equations (3) and (4) if and only if it satisfies (5).

Proof. Assume $x$ satisfies the equations (3) and (4) and such that the function $\sigma: t \longrightarrow\left(\frac{x(t)-\chi(t, x(t))}{f(t, x(t))}\right) \in$ $C_{\xi, \alpha}^{\xi}(J)$. We prove that $x$ is a solution to the equation (5). From the definition of the space $C_{\xi, \alpha}^{\xi}(J)$ and by using Lemma 2.3 and Definition 2.2, we have

$$
\left({ }^{\alpha} \mathbb{J}_{a^{+}}^{1-\xi} \sigma(\tau)\right)(t) \in C_{\xi, \alpha}(J)
$$

and

$$
{ }^{\alpha} \mathbb{D}_{a^{+}}^{\xi} \sigma(t)=\left(\delta_{\alpha}^{\alpha} \mathbb{J}_{a^{+}}^{1-\xi} \sigma(\tau)\right)(t) \in C_{\xi, \alpha}(J)
$$

We have

$$
\left({ }^{\alpha} \mathbb{J}_{a^{+}}^{1-\xi} \sigma(\tau)\right)(t) \in C_{\xi, \alpha}^{1}(J)
$$

Hence, Lemma 2.5 implies that

$$
\left({ }^{\alpha} \mathbb{J}_{a^{+}}^{\xi}{ }^{\alpha} \mathbb{D}_{a^{+}}^{\xi} \sigma(\tau)\right)(t)=\sigma(t)-\bar{\Psi}_{\xi}(t, a)\left({ }^{\alpha} \mathbb{J}_{a^{+}}^{1-\xi} \sigma(\tau)\right)(a), \text { for all } t \in(a, b] .
$$

Using Lemma 2.8 we have

$$
\begin{aligned}
\left({ }^{\alpha} \mathbb{J}_{a^{+}}^{\xi}{ }^{\alpha} \mathbb{D}_{a^{+}}^{\xi} \sigma(\tau)\right)(t) & =\left({ }^{\alpha} \mathbb{J}_{a^{+}}^{9}{ }^{\alpha} \mathbb{D}_{a^{+}}^{9, r} \sigma(\tau)\right)(t) \\
& =\left({ }^{\alpha} \mathbb{J}_{a^{+}}^{\vartheta} v(\tau)\right)(t) .
\end{aligned}
$$

Then,

$$
\frac{\chi(t)-\chi(t, x(t))}{f(t, x(t))}=\bar{\Psi}_{\xi}(t, a)\left({ }^{\alpha} \mathbb{J}_{a^{+}}^{1-\xi} \sigma(\tau)\right)(a)+\left({ }^{\alpha} \mathbb{J}_{a^{+}}^{g} v(\tau)\right)(t),
$$

which implies that

$$
x(t)=f(t, x(t))\left[\bar{\Psi}_{\xi}(t, a)\left({ }^{\alpha} \mathbb{J}_{a^{+}}^{1-\xi}\left(\frac{x(\tau)-\chi(\tau, x(\tau))}{f(\tau, x(\tau))}\right)\right)(a)+\left({ }^{\alpha} \mathbb{J}_{a^{+}}^{9} v(\tau)\right)(t)\right]+\chi(t, x(t)) .
$$

Next, we substitute $t=\epsilon_{i}$ into (6), then we multiply $c_{i}$ to both sides, we obtain

$$
c_{i}\left(\frac{x\left(\epsilon_{i}\right)-\chi\left(\epsilon_{i}, x\left(\epsilon_{i}\right)\right)}{f\left(\epsilon_{i}, x\left(\epsilon_{i}\right)\right)}\right)=c_{i} \bar{\Psi}_{\xi}\left(\epsilon_{i}, a\right)\left({ }^{\alpha} \mathbb{J}_{a^{+}}^{1-\xi} \sigma(\tau)\right)(a)+c_{i}\left({ }^{\alpha} \mathbb{J}_{a^{+}}^{g} v(\tau)\right)\left(\epsilon_{i}\right) .
$$

Then by using condition (4), we have

$$
\begin{aligned}
\left({ }^{\alpha} \mathbb{J}_{a^{+}}^{1-\xi} \sigma(\tau)\right)\left(a^{+}\right) & =\sum_{i=1}^{m} c_{i}\left(\frac{x\left(\epsilon_{i}\right)-\chi\left(\epsilon_{i}, x\left(\epsilon_{i}\right)\right)}{f\left(\epsilon_{i}, x\left(\epsilon_{i}\right)\right)}\right) \\
& =\left({ }^{\alpha} \mathbb{J}_{a^{+}}^{1-\xi} \sigma(\tau)\right)(a) \sum_{i=1}^{m} c_{i} \bar{\Psi}_{\xi}\left(\epsilon_{i}, a\right)+\sum_{i=1}^{m} c_{i}\left({ }^{\alpha} \mathbb{J}_{a^{+}}^{q} v(\tau)\right)\left(\epsilon_{i}\right),
\end{aligned}
$$

which implies

$$
\left({ }^{\alpha} \mathbb{J}_{a^{+}}^{1-\xi} \sigma(\tau)\right)\left(a^{+}\right)=\frac{\sum_{i=1}^{m} c_{i}\left({ }^{\alpha} \mathbb{J}_{a^{+}}^{g} v(\tau)\right)\left(\epsilon_{i}\right)}{1-\sum_{i=1}^{m} c_{i} \bar{\Psi}_{\xi}\left(\epsilon_{i}, a\right)}
$$


Substituting (7) into (6), we obtain (5).

Reciprocally, assume $x$ satisfies the equation (5) such that the function $\sigma: t \longrightarrow\left(\frac{x(t)-\chi(t, x(t))}{f(t, x(t))}\right) \in C_{\xi, \alpha}^{\xi}(J)$. Applying operator ${ }^{\alpha} \mathbb{D}_{a^{+}}^{\xi}$ on both sides of (5), and since $f(t, x(t)) \neq 0$ for all $t \in J$, then, from Lemma 2.8 we obtain

$$
\left({ }^{\alpha} \mathbb{D}_{a^{+}}^{\xi} \sigma(\tau)\right)(t)=\left({ }^{\alpha} \mathbb{D}_{a^{+}}^{r(1-9)} v(\tau)\right)(t) .
$$

Since $\sigma \in C_{\xi, \alpha}^{\xi}(J)$ we have ${ }^{\alpha} \mathbb{D}_{a^{+}}^{\xi} \sigma \in C_{\xi, \alpha}(J)$, then (8) implies that

$$
\left({ }^{\alpha} \mathbb{D}_{a^{+}}^{\xi} \sigma(\tau)\right)(t)=\left(\delta_{\alpha}^{\alpha} \mathbb{J}_{a^{+}}^{1-r(1-g)} v(\tau)\right)(t)=\left({ }^{\alpha} \mathbb{D}_{a^{+}}^{r(1-g)} v(\tau)\right)(t) \in C_{\xi, \alpha}(J) .
$$

As $v(\cdot) \in C_{\xi, \alpha}(J)$ and from Lemma 2.3, follows

$$
\left({ }^{\alpha} \mathbb{J}_{a^{+}}^{1-r(1-9)} v\right) \in C_{\xi, \alpha}(J) .
$$

From (9), (10) and by the definition of the space $C_{\xi, \alpha}^{n}(J)$, we obtain

$$
\left({ }^{\alpha} \mathbb{J}_{a^{+}}^{1-r(1-9)} v\right) \in C_{\xi, \alpha}^{1}(J) .
$$

Applying operator ${ }^{\alpha} \mathrm{J}_{a^{+}}^{r(1-9)}$ on both sides of (9) and using Lemma 2.5 and Property 2.7, we have

$$
\begin{aligned}
\left({ }^{\alpha} \mathbb{D}_{a^{+}}^{g, r} \sigma(\tau)\right)(t) & ={ }^{\alpha} \mathbb{J}_{a^{+}}^{r(1-9)}\left({ }^{\alpha} \mathbb{D}_{a^{+}}^{\xi} \sigma(\tau)\right)(t) \\
& =v(t)-\bar{\Psi}_{r(1-\vartheta)}(t, a)\left({ }^{\alpha} \mathbb{J}_{a^{+}}^{1-r(1-9)} v(\tau)\right)(a) \\
& =v(t),
\end{aligned}
$$

that is, (3) holds. Now, applying ${ }^{\alpha} \mathbb{J}_{a^{+}}^{1-\xi}$ on both sides of (5) we get

$$
\left({ }^{\alpha} \mathbb{J}_{a^{+}}^{1-\xi} \sigma(\tau)\right)(t)=\frac{\sum_{i=1}^{m} c_{i}\left({ }^{\alpha} \mathbb{J}_{a^{+}}^{9} v(\tau)\right)\left(\epsilon_{i}\right)}{1-\sum_{i=1}^{m} c_{i} \bar{\Psi}_{\xi}\left(\epsilon_{i}, a\right)}+\left({ }^{\alpha} \mathbb{J}_{a^{+}}^{1-\xi+9} v(\tau)\right)(t)
$$

Taking the limit $t \rightarrow a^{+}$of (11) we obtain

$$
\left(\alpha \mathbb{J}_{a^{+}}^{1-\xi}\left(\frac{x(\tau)-\chi(\tau, x(\tau))}{f(\tau, x(\tau))}\right)\right)\left(a^{+}\right)=\frac{\sum_{i=1}^{m} c_{i}\left({ }^{\alpha} \mathbb{J}_{a^{+}}^{9} v(\tau)\right)\left(\epsilon_{i}\right)}{1-\sum_{i=1}^{m} c_{i} \bar{\Psi}_{\xi}\left(\epsilon_{i}, a\right)} .
$$

Substituting $t=\epsilon_{i}$ into (5), we have

$$
\frac{\chi\left(\epsilon_{i}\right)-\chi\left(\epsilon_{i}, \chi\left(\epsilon_{i}\right)\right)}{f\left(\epsilon_{i}, \chi\left(\epsilon_{i}\right)\right)}=\bar{\Psi}_{\xi}\left(\epsilon_{i}, a\right) \frac{\sum_{i=1}^{m} c_{i}\left({ }^{\alpha} \mathbb{J}_{a^{+}}^{g} v(\tau)\right)\left(\epsilon_{i}\right)}{1-\sum_{i=1}^{m} c_{i} \bar{\Psi}_{\xi}\left(\epsilon_{i}, a\right)}+\left({ }^{\alpha} \mathbb{J}_{a^{+}}^{g} v(\tau)\right)\left(\epsilon_{i}\right) .
$$

Then, we have

$$
\sum_{i=1}^{m} c_{i}\left(\frac{x\left(\epsilon_{i}\right)-\chi\left(\epsilon_{i}, x\left(\epsilon_{i}\right)\right)}{f\left(\epsilon_{i}, x\left(\epsilon_{i}\right)\right)}\right)=\sum_{i=1}^{m} c_{i} \bar{\Psi}_{\xi}\left(\epsilon_{i}, a\right) \frac{\sum_{i=1}^{m} c_{i}\left({ }^{\alpha} \mathbb{J}_{a^{+}}^{9} v(\tau)\right)\left(\epsilon_{i}\right)}{1-\sum_{i=1}^{m} c_{i} \bar{\Psi}_{\xi}\left(\epsilon_{i}, a\right)}+\sum_{i=1}^{m} c_{i}\left({ }^{\alpha} \mathbb{J}_{a^{+}}^{9} v(\tau)\right)\left(\epsilon_{i}\right),
$$


thus,

$$
\sum_{i=1}^{m} c_{i}\left(\frac{\chi\left(\epsilon_{i}\right)-\chi\left(\epsilon_{i}, x\left(\epsilon_{i}\right)\right)}{f\left(\epsilon_{i}, x\left(\epsilon_{i}\right)\right)}\right)=\frac{\sum_{i=1}^{m} c_{i}\left({ }^{\alpha} \mathbb{J}_{a^{+}}^{9} v(\tau)\right)\left(\epsilon_{i}\right)}{1-\sum_{i=1}^{m} c_{i} \bar{\Psi}_{\xi}\left(\epsilon_{i}, a\right)} .
$$

From (12) and (13), we find that

$$
\left({ }^{\alpha} \mathbb{J}_{a^{+}}^{1-\xi}\left(\frac{x(\tau)-\chi(\tau, x(\tau))}{f(\tau, x(\tau))}\right)\right)\left(a^{+}\right)=\sum_{i=1}^{m} c_{i}\left(\frac{x\left(\epsilon_{i}\right)-\chi\left(\epsilon_{i}, x\left(\epsilon_{i}\right)\right)}{f\left(\epsilon_{i}, x\left(\epsilon_{i}\right)\right)}\right),
$$

which shows that the initial condition (4) is satisfied.

Conditions on $\varphi$ so that $\varphi(\cdot, x(\cdot), y(\cdot)) \in C_{\xi, \alpha}(J)$, for any $x, y \in C_{\xi, \alpha}(J)$ will be given below.

Lemma 3.2. Let $f \in C(J \times \mathbb{R}, \mathbb{R} \backslash\{0\}), \chi \in C(J \times \mathbb{R}, \mathbb{R})$. If the function $t \longrightarrow\left(\frac{x(t)-\chi(t, x(t))}{f(t, x(t))}\right) \in C_{\xi, \alpha}^{\xi}(J)$, then $x$ satisfies the problem (1) - (2) if and only if $x$ is the fixed point of the operator $\Im: C_{\xi, \alpha}(J) \rightarrow C_{\xi, \alpha}(J)$ defined by

$$
\Im x(t)=f(t, x(t))\left[K \bar{\Psi}_{\xi}(t, a) \sum_{i=1}^{m} c_{i}\left({ }^{\alpha} \mathbb{J}_{a^{+}}^{\vartheta} v(\tau)\right)\left(\epsilon_{i}\right)+\left({ }^{\alpha} \mathbb{J}_{a^{+}}^{\vartheta} v(\tau)\right)(t)\right]+\chi(t, x(t)),
$$

where $K=\left[1-\sum_{i=1}^{m} c_{i} \bar{\Psi}_{\xi}\left(\epsilon_{i}, a\right)\right]^{-1}$ and $v: J \rightarrow \mathbb{R}$ be a function satisfying the functional equation

$$
v(t)=\varphi(t, x(t), v(t))
$$

Since the functions $f$ and $\chi$ are continuous and $\varphi(\cdot, x(\cdot), y(\cdot)) \in C_{\xi, \alpha}(J)$, then, by Lemma 2.3, we have $\Im x \in$ $C_{\xi, \alpha}(J)$.

$(A \times 1) \varphi: J \times \mathbb{R}^{2} \rightarrow \mathbb{R}$ is continuous on $J$ and

$$
\varphi(\cdot, x(\cdot), y(\cdot)) \in C_{\xi, \alpha}^{r(1-\vartheta)}(J), \text { for any } x, y \in C_{\xi, \alpha}(J) .
$$

(Ax2)The functions $f: J \times \mathbb{R} \rightarrow \mathbb{R} \backslash\{0\}$ and $\chi: J \times \mathbb{R} \rightarrow \mathbb{R}$ are continuous and there exist two functions $p, q \in C(J,[0, \infty))$ such that

$$
|f(t, x)-f(t, \bar{x})| \leq p(t) \Psi_{\xi}(t, a)|x-\bar{x}|
$$

and

$$
|\chi(t, x)-\chi(t, \bar{x})| \leq q(t)|x-\bar{x}|
$$

for any $x, \bar{x} \in \mathbb{R}$ and $t \in(a, b]$.

(Ax3)There exists functions $\lambda_{1}, \lambda_{2}, \lambda_{3} \in C(J,[0, \infty))$ such that

$$
|\varphi(t, x, y)| \leq \lambda_{1}(t)+\lambda_{2}(t)|x|+\lambda_{3}(t)|y| \text { for } t \in(a, b] \text {, and } x, y \in \mathbb{R} \text {. }
$$

(Ax4)There exists a number $\ell>0$ such that

$$
\ell \geq \frac{f^{\star} M+\chi^{\star}}{1-p^{\star} M-q^{\star}}
$$

where

$$
\begin{gathered}
p^{\star}=\sup _{t \in J} p(t), \quad q^{\star}=\sup _{t \in J} q(t), \\
\lambda_{i}^{\star}=\sup _{t \in J} \lambda_{i}(t), i=1,2, \quad \lambda_{3}^{\star}=\sup _{t \in J} \lambda_{3}(t)<1, \\
f^{\star}=\sup _{t \in J}|f(t, 0)|, \quad \chi^{\star}=\sup _{t \in J} \Psi_{\xi}(t, a)|\chi(t, 0)|, \quad \Lambda:=\frac{\Psi_{\xi}(b, a) \lambda_{1}^{\star}+\lambda_{2}^{\star} \ell}{1-\lambda_{3}^{\star}}
\end{gathered}
$$


and

$$
M=\frac{\Lambda \alpha^{-\vartheta}}{\Gamma(\vartheta+\xi)}\left[|K| \alpha^{1-\xi} \sum_{i=1}^{m}\left|c_{i}\right|\left(\epsilon_{i}^{\alpha}-a^{\alpha}\right)^{\vartheta+\xi-1}+\Gamma(\xi)\left(b^{\alpha}-a^{\alpha}\right)^{\vartheta}\right]
$$

Theorem 3.3. Assume (Ax1)-(Ax4) hold. If

$$
\max \left\{p^{\star} M, p^{\star} \Psi_{\xi}(b, a) M\right\}+q^{\star}<1,
$$

then the problem (1)-(2) has at least one solution in $C_{\xi, \alpha}(J)$.

Proof. We define a subset $\Omega$ of $C_{\xi, \alpha}(J)$ by

$$
\Omega=\left\{x \in C_{\xi, \alpha}(J):\|x\|_{\xi, \alpha} \leq \ell\right\} .
$$

We consider the operator $\Im$ defined in (14), and define three operators $\mathcal{S}, \mathcal{N}: C_{\xi, \alpha}(J) \rightarrow C_{\xi, \alpha}(J), \mathcal{T}: \Omega \rightarrow$ $C_{\xi, \alpha}(J)$ by

$$
\begin{aligned}
& (\mathcal{S} x)(t)=f(t, x(t)), \quad t \in(a, b], \\
& (\mathcal{N} x)(t)=\chi(t, x(t)), \quad t \in(a, b],
\end{aligned}
$$

and

$$
(\mathcal{T} X)(t)=K \bar{\Psi}_{\xi}(t, a) \sum_{i=1}^{m} c_{i}\left({ }^{\alpha} \mathbb{J}_{a^{+}}^{\vartheta} v(\tau)\right)\left(\epsilon_{i}\right)+\left({ }^{\alpha} \mathbb{J}_{a^{+}}^{\vartheta} v(\tau)\right)(t), \quad t \in(a, b] .
$$

Then we get $\Im x=S \mathcal{S} x x+\mathcal{N} x$.

Step 1: The operators $\mathcal{S}$ and $\mathcal{N}$ are Lipschitzian on $C_{\xi, \alpha}(J)$. Let $x, y \in C_{\xi, \alpha}(J)$ and $t \in(a, b]$. Then by $(A x 2)$ we have

$$
\begin{aligned}
\left|((\mathcal{S} x)(t)-(\mathcal{S} y)(t)) \Psi_{\xi}(t, a)\right| & \leq \Psi_{\xi}(t, a)|f(t, x)-f(t, y)|, \\
& \leq p(t) \Psi_{\xi}(t, a)\|x-y\|_{\xi, \alpha}, \\
& \leq p^{\star} \Psi_{\xi}(b, a)\|x-y\|_{\xi, \alpha},
\end{aligned}
$$

then for each $t \in(a, b]$ we obtain

$$
\|\mathcal{S} x-\mathcal{S} y\|_{\xi, \alpha} \leq p^{\star} \Psi_{\xi}(b, a)\|x-y\|_{\xi, \alpha} .
$$

Also, for each $t \in(a, b]$ we have

$$
\begin{aligned}
\left|((\mathcal{N} x)(t)-(\mathcal{N} y)(t)) \Psi_{\xi}(t, a)\right| & \leq \Psi_{\xi}(t, a)|\chi(t, x(t))-\chi(t, y(t))|, \\
& \leq q(t)\|x-y\|_{\xi, \alpha}, \\
& \leq q^{\star}\|x-y\|_{\xi, \alpha},
\end{aligned}
$$

then,

$$
\|\mathcal{N} x-\mathcal{N} y\|_{\xi, \alpha} \leq q^{\star}\|x-y\|_{\xi, \alpha} .
$$

Step 2: The operator $\mathcal{T}$ is completely continuous on $\Omega$.

We firstly show that the operator $\mathcal{T}$ is continuous on $\Omega$. Let $\left\{x_{n}\right\}$ be sequence in $\Omega$ such that $x_{n} \rightarrow x$ in $\Omega$. Let $x, y \in C_{\xi, \alpha}(J)$.

Then for each $t \in(a, b]$, we have

$$
\left.\mid\left(\mathcal{T} x_{n}\right)(t)-(\mathcal{T} x)(t)\right) \Psi_{\xi}(t, a)\left|\leq \frac{|K|}{\Gamma(\xi)} \sum_{i=1}^{m}\right| c_{i} \mid\left({ }^{\alpha} \mathbb{J}_{a^{+}}^{\vartheta}\left|v_{n}(\tau)-v(\tau)\right|\right)\left(\epsilon_{i}\right)
$$




$$
+\quad \Psi_{\xi}(t, a)\left({ }^{\alpha} \mathbb{J}_{a^{+}}^{9}\left|v_{n}(\tau)-v(\tau)\right|\right)(t),
$$

where $v_{n}, v \in C_{\xi, \alpha}(J)$ such that

$$
\begin{array}{r}
v_{n}(t)=\varphi\left(t, x_{n}(t), v_{n}(t)\right), \\
v(t)=\varphi(t, x(t), v(t)) .
\end{array}
$$

Since $x_{n} \rightarrow x$ and $\varphi$ is a continuous function on $J$ then we get $v_{n}(t) \rightarrow v(t)$ as $n \rightarrow \infty$ for each $t \in(a, b]$, so by Lebesgue dominated convergence theorem, we have

$$
\left\|\mathcal{T} x_{n}-\mathcal{T} x\right\|_{c_{\xi, \alpha}} \rightarrow 0 \text { as } n \rightarrow \infty
$$

Then $\mathcal{T}$ is continuous.

Next we prove that $\mathcal{T}(\Omega)$ is uniformly bounded on $C_{\xi, \alpha}(J)$. Let any $x \in \Omega$. By $(A x 3)$, we have for each $t \in(a, b]$

$$
\begin{aligned}
\left|\Psi_{\xi}(t, a) v(t)\right| & =\left|\Psi_{\xi}(t, a) \varphi(t, x(t), v(t))\right| \\
& \leq \Psi_{\xi}(t, a)\left(\lambda_{1}(t)+\lambda_{2}(t)|x(t)|+\lambda_{3}(t)|v(t)|\right) \\
& \leq \Psi_{\xi}(b, a) \lambda_{1}^{\star}+\lambda_{2}^{\star} \ell+\lambda_{3}^{\star}\left|\Psi_{\xi}(t, a) v(t)\right| .
\end{aligned}
$$

Hence

$$
\left|\Psi_{\xi}(t, a) v(t)\right| \leq \frac{\Psi_{\xi}(b, a) \lambda_{1}^{\star}+\lambda_{2}^{\star} \ell}{1-\lambda_{3}^{\star}}
$$

Then, we have

$$
\sup _{t \in(a, b]}\left|\Psi_{\xi}(t, a) v(t)\right| \leq \frac{\Psi_{\xi}(b, a) \lambda_{1}^{\star}+\lambda_{2}^{\star} \ell}{1-\lambda_{3}^{\star}}:=\Lambda .
$$

For $t \in(a, b]$, by (19) we have

$$
\begin{aligned}
& \left|\Psi_{\xi}(t, a)(\mathcal{T} x)(t)\right| \\
\leq & \frac{|K|}{\Gamma(\xi)} \sum_{i=1}^{m}\left|c_{i}\right|\left({ }^{\alpha} \mathbb{J}_{a^{+}}^{9}|v(\tau)|\right)\left(\epsilon_{i}\right)+\Psi_{\xi}(t, a)\left({ }^{\alpha} \mathbb{J}_{a^{+}}^{9}|v(\tau)|\right)(t) \\
\leq & \Lambda|K| \sum_{i=1}^{m}\left|c_{i}\right|\left({ }^{\alpha} \mathbb{J}_{a^{+}}^{9} \bar{\Psi}_{\xi}(\tau, a)\right)\left(\epsilon_{i}\right)+\Lambda \Psi_{\xi}(t, a) \Gamma(\xi)\left({ }^{\alpha} \mathbb{J}_{a^{+}}^{9} \bar{\Psi}_{\xi}(\tau, a)\right)(t) \\
\leq & \Lambda|K| \sum_{i=1}^{m}\left|c_{i}\right| \bar{\Psi}_{9+\xi}\left(\epsilon_{i}, a\right)+\Lambda \Psi_{\xi}(t, a) \Gamma(\xi) \bar{\Psi}_{9+\xi}(t, a) \\
\leq & \frac{\Lambda|K|}{\Gamma(\vartheta+\xi)} \sum_{i=1}^{m}\left|c_{i}\right|\left(\frac{\epsilon_{i}^{\alpha}-a^{\alpha}}{\alpha}\right)^{9+\xi-1}+\frac{\Lambda \Gamma(\xi)}{\Gamma(\vartheta+\xi)}\left(\frac{b^{\alpha}-a^{\alpha}}{\alpha}\right)^{\vartheta} .
\end{aligned}
$$

Then we obtain

$$
\|\mathcal{T} X\|_{C_{\xi, \alpha}} \leq M .
$$

This prove that the operator $\mathcal{T}$ is uniformly bounded on $\Omega$. Next we prove that the operator $\mathcal{T} \Omega$ is equicontinuous. We take $x \in \Omega$ and $a<\varepsilon_{1}<\varepsilon_{2} \leq b$. Then,

$$
\begin{aligned}
& \leq \mid \begin{array}{l}
\Psi_{\xi}\left(\varepsilon_{1}, a\right)(\mathcal{T} x)\left(\varepsilon_{1}\right)-\Psi_{\xi}\left(\varepsilon_{2}, a\right)(\mathcal{T} x)\left(\varepsilon_{2}\right) \mid \\
\Psi_{\xi}\left(\varepsilon_{1}, a\right)\left({ }^{\alpha} \mathbb{J}_{a^{+}}^{\vartheta} v(\tau)\right)\left(\varepsilon_{1}\right)-\Psi_{\xi}\left(\varepsilon_{2}, a\right)\left({ }^{\alpha} \mathbb{J}_{a^{+}}^{g} v(\tau)\right)\left(\varepsilon_{2}\right) \mid
\end{array} \\
& \leq \int_{a}^{\varepsilon_{1}}\left|\Psi_{\xi}\left(\varepsilon_{1}, a\right) \bar{\Psi}_{\vartheta}\left(\varepsilon_{1}, \tau\right)-\Psi_{\xi}\left(\varepsilon_{2}, a\right) \bar{\Psi}_{\vartheta}\left(\varepsilon_{2}, \tau\right)\right|\left|\tau^{\alpha-1} v(\tau)\right| d \tau \\
& +\quad \Psi_{\xi}\left(\varepsilon_{2}, a\right)\left({ }^{\alpha} \mathbb{J}_{\mathcal{E}_{1}^{+}}^{9}|v(\tau)|\right)\left(\varepsilon_{2}\right) \text {. }
\end{aligned}
$$


Then we have for each $t \in(a, b]$

$$
\begin{aligned}
& \left|\Psi_{\xi}\left(\varepsilon_{1}, a\right)(\mathcal{T} x)\left(\varepsilon_{1}\right)-\Psi_{\xi}\left(\varepsilon_{2}, a\right)(\mathcal{T} x)\left(\varepsilon_{2}\right)\right| \\
\leq & \Lambda \Gamma(\xi) \int_{a}^{\varepsilon_{1}} \tau^{\alpha-1}\left|\Psi_{\xi}\left(\varepsilon_{1}, a\right) \bar{\Psi}_{\vartheta}\left(\varepsilon_{1}, \tau\right)-\Psi_{\xi}\left(\varepsilon_{2}, a\right) \bar{\Psi}_{\vartheta}\left(\varepsilon_{2}, \tau\right)\right| \bar{\Psi}_{\xi}(\tau, a) d \tau, \\
& +\Lambda \Gamma(\xi) \Psi_{\xi}\left(\varepsilon_{2}, a\right) \bar{\Psi}_{\vartheta+\xi}\left(\varepsilon_{2}, \varepsilon_{1}\right) .
\end{aligned}
$$

Note that

$$
\left|\Psi_{\xi}\left(\varepsilon_{1}, a\right)(\mathcal{T} x)\left(\varepsilon_{1}\right)-\Psi_{\xi}\left(\varepsilon_{2}, a\right)(\mathcal{T} x)\left(\varepsilon_{2}\right)\right| \rightarrow 0 \text { as } \varepsilon_{1} \rightarrow \varepsilon_{2} \text {. }
$$

This proves that $\mathcal{T} \Omega$ is equicontinuous on $J$. Therefore by the Arzelà-Ascoli theorem, $\mathcal{T}$ is completely continuous on $\Omega$.

Step 3: The third hypothesis of Lemma 2.9 is satisfied.

Let $x \in C_{\xi, \alpha}(J)$ and $y \in \Omega$ be arbitrary such that $x=\mathcal{S} x \mathcal{T} y+\mathcal{N} x$ and $\tilde{v} \in C_{\xi, \alpha}(J)$ with

$$
\tilde{v}(t)=\varphi(t, y(t), \tilde{v}(t))
$$

Then, for $t \in(a, b]$ we have

$$
\begin{aligned}
& \left|\Psi_{\xi}(t, a) x(t)\right| \\
= & \left|\Psi_{\xi}(t, a)(\mathcal{S} x \mathcal{T} y)(t)+\Psi_{\xi}(t, a)(\mathcal{N} x)(t)\right| \\
\leq & \Psi_{\xi}(t, a)|(\mathcal{S} x)(t)||(\mathcal{T} y)(t)|+\left|\Psi_{\xi}(t, a)(\mathcal{N} x)(t)\right| \\
\leq & |f(t, x(t))|\left[\frac{|K|}{\Gamma(\xi)} \sum_{i=1}^{m}\left|c_{i}\right|\left({ }^{\alpha} \mathbb{J}_{a^{+}}^{\vartheta}|\tilde{v}(\tau)|\right)\left(\epsilon_{i}\right)+\Psi_{\xi}(t, a)\left({ }^{\alpha} \mathbb{J}_{a^{+}}^{\vartheta}|\tilde{v}(\tau)|\right)(t)\right] \\
& +\Psi_{\xi}(t, a)|\chi(t, x(t))| \\
\leq & M(|f(t, x(t))-f(t, 0)|+|f(t, 0)|)+\Psi_{\xi}(t, a)(|\chi(t, x(t))-\chi(t, 0)|+|\chi(t, 0)|) \\
\leq & M\left(p^{\star}\|x\|_{C_{\xi, \alpha}}+f^{\star}\right)+q^{\star}\|x\|_{C_{\xi, \alpha}}+\chi^{\star},
\end{aligned}
$$

then,

$$
\|x\|_{C_{\xi, \alpha}}=\frac{f^{\star} M+\chi^{\star}}{1-p^{\star} M-q^{\star}} \leq \ell .
$$

Then $x \in \Omega$, thus the third hypothesis of Lemma 2.9 is satisfied.

Step 4: The fourth hypothesis of Lemma 2.9 is satisfied. We show that $p^{\star} \Psi_{\xi}(b, a) L+q^{\star}<1$, where

$$
L=\|\mathcal{T}(\Omega)\|_{C_{\xi, \alpha}}=\sup \left\{\|\mathcal{T} y\|_{C_{\xi, \alpha}}: y \in \Omega\right\} .
$$

Since $L \leq M$, we have

$$
p^{\star} \Psi_{\xi}(b, a) L+q^{\star} \leq p^{\star} \Psi_{\xi}(b, a) M+q^{\star}<1 .
$$

That is, the last hypothesis of Lemma 2.9 is satisfied. Thus, the operator equation $\Im x=\mathcal{S} x \mathcal{T} x+\mathcal{N} x=x$ has at least one solution $x^{\star} \in C_{\xi, \alpha}$, which is a fixed point for the operator $\Im$.

Step 5: We prove that for such fixed point $x^{\star} \in C_{\xi, \alpha}(J)$, the function $\sigma: t \rightarrow \frac{x^{\star}(t)-\chi\left(t, x^{\star}(t)\right)}{f\left(t, x^{\star}(t)\right)}$ is in $C_{\xi, \alpha}^{\xi}(J)$. Since $x^{\star}$ is a fixed point of operator $\Im$ in $C_{\xi, \alpha}(J)$, then for each $t \in(a, b]$, we have

$$
\Im \chi^{\star}(t)=f\left(t, x^{\star}(t)\right)\left[K \bar{\Psi}_{\xi}(t, a) \sum_{i=1}^{m} c_{i}\left({ }^{\alpha} \mathbb{J}_{a^{+}}^{\vartheta} v(\tau)\right)\left(\epsilon_{i}\right)+\left({ }^{\alpha} \mathbb{J}_{a^{+}}^{\vartheta} v(\tau)\right)(t)\right]+\chi\left(t, x^{\star}(t)\right) .
$$

where $v \in C_{\xi, \alpha}(J)$ such that

$$
v(t)=\varphi\left(t, x^{\star}(t), v(t)\right)
$$


Applying ${ }^{\alpha} \mathbb{D}_{a^{+}}^{\xi}$ to both sides of 20, and by Lemma 2.8, we have

$$
\begin{aligned}
\alpha \mathbb{D}_{a^{+}}^{\xi}\left(\frac{\chi^{\star}(t)-\chi\left(t, \chi^{\star}(t)\right)}{f\left(t, x^{\star}(t)\right)}\right) & =\left({ }^{\alpha} \mathbb{D}_{a^{+}}^{\xi} \alpha \mathbb{J}_{a^{+}}^{g} v(\tau)\right)(t) \\
& =\left({ }^{\alpha} \mathbb{D}_{a^{+}}^{r(1-g)} v(\tau)\right)(t) .
\end{aligned}
$$

Since $\xi \geq \vartheta$, by (Ax1), the right hand side is in $C_{\xi, \alpha}(J)$ and thus ${ }^{\alpha} \mathbb{D}_{\alpha^{+}}^{\xi} \sigma \in C_{\xi, \alpha}(J)$. It is clear that $\sigma \in C_{\xi, \alpha}(J)$, since $f \in C(J \times \mathbb{R} \rightarrow \mathbb{R} \backslash\{0\})$ and $\chi \in C(J \times \mathbb{R} \rightarrow \mathbb{R})$, then $\sigma \in C_{\xi, \alpha}^{\xi}(J)$. As a consequence of Steps 1-5 with Lemma 3.2, we can conclude that the problem (1) - (2) has at least a solution in $C_{\xi, \alpha}(J)$. This completes the proof of Theorem 3.3.

\section{Examples}

Example 4.1. Consider the problem

$$
\begin{gathered}
{ }^{1} \mathbb{D}_{1^{+}}^{\frac{1}{2}, 0}\left(\frac{x(t)-\chi(t, x(t))}{f(t, x(t))}\right)=\frac{\sqrt{t-1}\left(x(t)+{ }^{1} \mathbb{D}_{1^{+}}^{\frac{1}{2}, 0}\left(\frac{x(t)-\chi(t, x(t))}{f(t, x(t))}\right)+1\right)}{111 e^{-t+2}(1+\sqrt{t-1}|x(t)|)}, \text { for each } t \in(1,2], \\
\left({ }^{1} \mathbb{J}_{1^{+}}^{\frac{1}{2}}\left(\frac{x(\tau)-\chi(\tau, x(\tau))}{f(\tau, x(\tau))}\right)\right)\left(1^{+}\right)=2\left(\frac{x\left(\frac{3}{2}\right)-\chi\left(\frac{3}{2}, x\left(\frac{3}{2}\right)\right)}{f\left(\frac{3}{2}, x\left(\frac{3}{2}\right)\right)}\right),
\end{gathered}
$$

where $I=[1,2], a=1, b=2$ and

$$
f(t, x(t))=\frac{(t-1)(|x(t)|+1)}{41 e^{-t+4}}, t \in I, x \in C_{\frac{1}{2}, 1}(I),
$$

and

$$
\chi(t, x(t))=\frac{\sqrt{t-1} \ln (2) x(t)}{33 e^{3} \sqrt{6-t}}+\frac{1}{55 e^{-t+2}}, t \in I, x \in C_{\frac{1}{2}, 1}(I)
$$

Set

$$
\varphi(t, x, y)=\frac{\sqrt{t-1}(x+y+1)}{111 e^{-t+2}(1+|x| \sqrt{t-1})}, t \in I, x, y \in \mathbb{R} .
$$

We have

$$
C_{\xi, \alpha}^{r(1-9)}(I)=C_{\frac{1}{2}, 1}^{0}(I)=\{v:(1,2] \rightarrow \mathbb{R}: t \rightarrow(\sqrt{t-1}) v(t) \in C(I, \mathbb{R})\},
$$

with $\xi=\vartheta=\frac{1}{2}, \alpha=1, r=0$. Clearly, the continuous function $\varphi \in C_{\frac{1}{2}, 1}^{0}(I)$. Hence the condition (Ax1) is satisfied. For each $x, \bar{x} \in \mathbb{R}$ and $t \in I$, we have

$$
|f(t, x)-f(t, \bar{x})| \leq \frac{t-1}{41 e^{-t+4}}|x-\bar{x}|,
$$

and

$$
|\chi(t, x)-\chi(t, \bar{x})| \leq \frac{\sqrt{t-1} \ln (2)}{33 e^{3} \sqrt{6-t}}|x-\bar{x}| .
$$

Hence condition ( $\mathrm{Ax} 2)$ is satisfied with

$$
p(t)=\frac{\sqrt{t-1}}{41 e^{-t+4}}, \text { and } q(t)=\frac{\sqrt{t-1} \ln (2)}{33 e^{3} \sqrt{6-t}},
$$

so we have

$$
p^{\star}=\frac{1}{41 e^{2}} \text {, and } q^{\star}=\frac{\ln (2)}{66 e^{3}} \text {. }
$$

Let $x, y \in \mathbb{R}$. Then we have

$$
|\varphi(t, x, y)| \leq \frac{\sqrt{t-1}}{111 e^{-t+2}}(|x|+|y|+1), t \in I,
$$


and so the condition (Ax3) is satisfied with

$$
\lambda_{1}(t)=\lambda_{2}(t)=\lambda_{3}(t)=\frac{\sqrt{t-1}}{111 e^{-t+2}},
$$

and

$$
\lambda_{1}^{\star}=\lambda_{2}^{\star}=\lambda_{3}^{\star}=\frac{1}{111}
$$

Setting

$$
f^{\star}=\frac{1}{41 e^{2}}, \quad \chi^{\star}=\frac{1}{55}, \quad K=\frac{\sqrt{\pi}}{\sqrt{\pi}-2 \sqrt{2}}
$$

and

$$
\Lambda=\frac{1+\ell}{110} .
$$

Using these values, it follows by (15) and (16) that the constant $\ell$ satisfies the inequality

$$
0.01835103855 \leq \ell<\frac{4510 e^{2}\left(66 e^{3}-\ln (2)\right)(2 \sqrt{2}-\sqrt{\pi})}{66 e^{3}(2 \sqrt{\pi}+2 \sqrt{2 \pi}-\pi)}-1 \approx 6492.31995291809 .
$$

Thus, all the conditions of Theorem 3.3 are satisfied. Hence, our problem (21)-(22) has at least in $C_{\frac{1}{2}, 1}(I)$.

Example 4.2. Consider the problem

$$
\begin{gathered}
1 \mathbb{D}_{1^{+}}^{\frac{1}{2}, 0}\left(\frac{x(t)-\chi(t, x(t))}{f(t, x(t))}\right)=\frac{\sqrt{t-1}\left(x(t)+{ }^{1} \mathbb{D}_{1^{+}}^{\frac{1}{2}, 0}\left(\frac{x(t)-\chi(t, x(t))}{f(t, x(t))}\right)+1\right)}{111 e^{-t+2}(1+\sqrt{t-1}|x(t)|)}, \text { for each } t \in(1,2], \\
\left({ }^{1} \mathbb{J}_{1^{+}}^{\frac{1}{2}}\left(\frac{x(\tau)-\chi(\tau, x(\tau))}{f(\tau, x(\tau))}\right)\right)\left(1^{+}\right)=3\left(\frac{x\left(\frac{5}{4}\right)-\chi\left(\frac{5}{4}, x\left(\frac{5}{4}\right)\right)}{f\left(\frac{5}{4}, x\left(\frac{5}{4}\right)\right)}\right)+2\left(\frac{x\left(\frac{4}{3}\right)-\chi\left(\frac{4}{3}, x\left(\frac{4}{3}\right)\right)}{f\left(\frac{4}{3}, x\left(\frac{4}{3}\right)\right)}\right),
\end{gathered}
$$

where $I=[1,2], a=1, b=2$ and

$$
f(t, x(t))=\frac{(t-1)(|x(t)|+1)}{41 e^{-t+4}}, t \in I, x \in C_{\frac{1}{2}, 1}(I),
$$

and

$$
\chi(t, x(t))=\frac{\sqrt{t-1} \ln (2) x(t)}{33 e^{3} \sqrt{6-t}}+\frac{1}{55 e^{-t+2}}, t \in I, x \in C_{\frac{1}{2}, 1}(I) .
$$

Set

$$
\varphi(t, x, y)=\frac{\sqrt{t-1}(x+y+1)}{111 e^{-t+2}(1+|x| \sqrt{t-1})}, t \in I, x, y \in \mathbb{R}
$$

We have

$$
C_{\xi, \alpha}^{r(1-9)}(I)=C_{\frac{1}{2}, 1}^{0}(I)=\{v:(1,2] \rightarrow \mathbb{R}: t \rightarrow(\sqrt{t-1}) v(t) \in C(I, \mathbb{R})\},
$$

with $\xi=\vartheta=\frac{1}{2}, \alpha=1, r=0$. Clearly, the continuous function $\varphi \in C_{\frac{1}{2}, 1}^{0}(I)$. Hence the condition (Ax1) is satisfied. For each $x, \bar{x} \in \mathbb{R}$ and $t \in I$, we have

$$
|f(t, x)-f(t, \bar{x})| \leq \frac{t-1}{41 e^{-t+4}}|x-\bar{x}|,
$$

and

$$
|\chi(t, x)-\chi(t, \bar{x})| \leq \frac{\sqrt{t-1} \ln (2)}{33 e^{3} \sqrt{6-t}}|x-\bar{x}| .
$$

Hence condition (Ax2) is satisfied with

$$
p(t)=\frac{\sqrt{t-1}}{41 e^{-t+4}}, \text { and } q(t)=\frac{\sqrt{t-1} \ln (2)}{33 e^{3} \sqrt{6-t}},
$$

so we have

$$
p^{\star}=\frac{1}{41 e^{2}} \text {, and } q^{\star}=\frac{\ln (2)}{66 e^{3}} \text {. }
$$


Let $x, y \in \mathbb{R}$. Then we have

$$
|\varphi(t, x, y)| \leq \frac{\sqrt{t-1}}{111 e^{-t+2}}(|x|+|y|+1), t \in I,
$$

and so the condition $(\mathrm{Ax} 3)$ is satisfied with

$$
\lambda_{1}(t)=\lambda_{2}(t)=\lambda_{3}(t)=\frac{\sqrt{t-1}}{111 e^{-t+2}},
$$

and

$$
\lambda_{1}^{\star}=\lambda_{2}^{\star}=\lambda_{3}^{\star}=\frac{1}{111} .
$$

Setting

$$
m=2, f^{\star}=\frac{1}{41 e^{2}}, \chi^{\star}=\frac{1}{55}, K=\frac{\sqrt{\pi}}{\sqrt{\pi}-2 \sqrt{3}-6},
$$

and

$$
\Lambda=\frac{1+\ell}{110} .
$$

Same as the last example, it follows by (15) and (16) that the constant $\ell$ satisfies the inequality

$$
0.0183510386 \leq \ell<\frac{4510 e^{2}\left(66 e^{3}-\ln (2)\right)(2 \sqrt{3}-\sqrt{\pi}+6)}{66 e^{3}(11 \sqrt{\pi}+2 \sqrt{3 \pi}-\pi)}-1 \approx 11387 .
$$

Then the problem (23)-(24) has at least one solution in $C_{\frac{1}{2}, 1}(I)$.

Acknowledgements: We are grateful to the referees for their careful reading of the paper and for their remarks. The research of Juan J. Nieto has been partially supported by the Agencia Estatal de Investigación (AEI) of Spain, co-financed by the European Fund for Regional Development (FEDER) corresponding to the 20142020 multiyear financial framework, project MTM2016-75140-P and by Xunta de Galicia under grant ED431C 2019/02.

Conflict of interest: On behalf of all authors, the corresponding author states that there is no conflict of interest.

Data Availability Statement: Data sharing is not applicable to this article as no datasets were generated or analysed during the current study.

\section{References}

[1] S. Abbas, M. Benchohra, J. R. Graef and J. Henderson, Implicit Differential and Integral Equations: Existence and stability, Walter de Gruyter, London, 2018.

[2] S. Abbas, M. Benchohra, J. E. Lazreg, A. Alsaedi and Y. Zhou, Existence and Ulam stability for fractional differential equations of Hilfer-Hadamard type, Adv. Difference Equ. (2017) $14 \mathrm{p}$.

[3] S. Abbas, M. Benchohra, J. E. Lazreg and G. N'Guérékata, Hilfer and Hadamard functional random fractional differential inclusions, Cubo 19 (2017), 17-38.

[4] S. Abbas, M. Benchohra, J. E. Lazreg and Y. Zhou, A survey on Hadamard and Hilfer fractional differential equations: analysis and stability, Chaos Solitons Fractals 102 (2017), 47-71.

[5] S. Abbas, M. Benchohra and G. M. N’Guérékata, Topics in Fractional Differential Equations, Springer-Verlag, New York, 2012.

[6] S. Abbas, M. Benchohra and G. M. N'Guérékata, Advanced Fractional Differential and Integral Equations, Nova Science Publishers, New York, 2014.

[7] B. Ahmad, A. Alsaedi, S. K. Ntouyas and J. Tariboon, Hadamard-type Fractional Differential Equations, Inclusions and Inequalities. Springer, Cham, 2017.

[8] B. Ahmad and S. K. Ntouyas, Fractional differential inclusions with fractional separated boundary conditions, Fract. Calc. Appl. Anal. 15 (2012), 362-382.

[9] B. Ahmad, S. K. Ntouyas, Initial value problems for hybrid Hadamard fractional differential equations. Electron. J. Differential Equations 2014 (2014), 161.

[10] R. Almeida, A. B. Malinowska and T. Odzijewicz, Fractional differential equations with dependence on the CaputoKatugampola derivative, J. Comput. Nonlinear Dynam. 11 (6) (2016), 1-11. 
[11] D. Baleanu, Z. B. Güvenç, and J. A. T. Machado New Trends in Nanotechnology and Fractional Calculus Applications, Springer, New York, 2010.

[12] Z. Baitiche, K. Guerbati, M. Benchohra, Y. Zhou, Boundary value problems for hybrid Caputo fractional differential equations. Mathematics 2019 , 7, 282.

[13] M. Benchohra, S. Bouriah, J. J. Nieto, Terminal value problem for differential equations with Hilfer-Katugampola fractional derivative. Symmetry 2019, 11, 672.

[14] M. Benchohra, S. Hamani, S. K. Ntouyas, Boundary value problems for differential equations with fractional order. Surv. Math. Appl. 2008, 3, 1-12.

[15] M. Benchohra and J. E. Lazreg, Existence and Ulam stability for Nonlinear implicit fractional differential equations with Hadamard derivative, Stud. Univ. Babes-Bolyai Math. 62 (2017), 27-38.

[16] C. Derbazi, H. Hammouche, M. Benchohra, and Y. Zhou. Fractional hybrid differential equations with three-point boundary hybrid conditions. Adv Difference Equ. 2019 (2019), 125.

[17] B. C. Dhage, A fixed point theorem in Banach algebras with applications to functional integral equations. Kyungpook Math. J. 44 (2004), 145-155.

[18] B. C. Dhage, On a fixed point theorem in Banach algebras with applications. Appl. Math. Lett. 18 (2005), 273-280.

[19] K. Hilal, A. Kajouni, Boundary value problems for hybrid differential equations with fractional order. Adv Difference Equ. 2015, (2015), 183.

[20] U. Katugampola, A new approach to a generalized fractional integral, Appl. Math. Comput. 218 (2011), 860-865.

[21] K. D. Kucche, J. P. Kharade, Global existence and Ulam-Hyers stability of $\psi$-Hilfer fractional differential equations, Kyungpook Math. J. 60 (3) (2020), 647-671.

[22] K. N. Nabi, H. Abboubakar, P. Kumar, Forecasting of COVID-19 pandemic: From integer derivatives to fractional derivatives, Chaos, Solitons and Fractals (2020) 141,110283.

[23] F. Ndaïrou, I. Area, J. J. Nieto, D. F. M. Torres, Mathematical modeling of COVID-19 transmission dynamics with a case study of Wuhan, Chaos, Solitons Fractals (2020) 135, 109846.

[24] D. S. Oliveira, E. Capelas de Oliveira, Hilfer-Katugampola fractional derivatives, Comput. Appl. Math. 37 (2018), no. 3, 36723690.

[25] A. T. Sahlol, D. Yousri, A. A. Ewees, M. A. A. Al-qaness, R. Damasevicius, M. Abd Elaziz, COVID-19 image classification using deep features and fractional-order marine predators algorithm, Scientific Reports 10 (1) (2020),15364.

[26] A. Salim, M. Benchohra, E. Karapinar and J. E. Lazreg, Existence and Ulam stability for impulsive generalized Hilfer-type fractional differential equations, Adv. Difference Equ. (620) (2020), 1-21.

[27] A. Salim, M. Benchohra, J. E. Lazreg and J. Henderson, Nonlinear implicit generalized Hilfer-type fractional differential equations with non-instantaneous impulses in Banach spaces, Adv. Theor. Nonl. Anal. Appl. 4 (4) (2020), 332-348.

[28] A. Salim, M. Benchohra, J. R. Graef and J. E. Lazreg, Boundary value problem for fractional generalized Hilfer-type fractional derivative with non-instantaneous impulses, Fractal Fract. (2021) N 5 (1), 1-21.

[29] H. M. Srivastava, Fractional-order derivatives and integrals: Introductory overview and recent developments, Kyungpook Math. J. 60 (1) (2020), 73-116.

[30] J. R. Wang, Y. R. Zhang, Nonlocal initial value problems for differential equations with Hilfer fractional derivative. Appl. Math. Comput. 266 (2015), 850-859.

[31] Y. Zhao, S. Sun, Z. Han, Q. Li, Theory of fractional hybrid differential equations. Comput. Math. Appl. 62 (2011), $1312-1324$.

[32] Y. Zhou, J. R. Wang, L. Zhang, Basic Theory of Fractional Differential Equations. Second edition. World Scientific Publishing Co. Pte. Ltd., Hackensack, NJ, 2017. 